LA-14033

Approved for public release;

distribution is unlimited.

\title{
The Induction of Chaos in
}

Electronic Circuits

Final Report — October 1, 2001 
Los Alamos National Laboratory, an affirmative action/ equal opportunity employer, is operated by the University of California for the United States Department of Energy under contract W-7405-ENG-36.

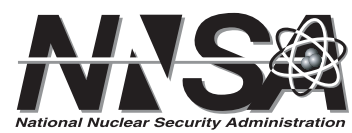

This report was prepared as an account of work sponsored by an agency of the United States Government. Neither the Regents of the University of California, the United States Government nor any agency thereof, nor any of their employees make any warranty, express or implied, or assume any legal liability or responsibility for the accuracy, completeness, or usefulness of any information, apparatus, product, or process disclosed, or represent that its use would not infringe privately owned rights. Reference herein to any specific commercial product, process, or service by trade name, trademark, manufacturer, or otherwise does not necessarily constitute or imply its endorsement, recommendation, or favoring by the Regents of the University of California, the United States Government, or any agency thereof. The views and opinions of authors expressed herein do not necessarily state or reflect those of the Regents of the University of California, the United States Government, or any agency thereof. Los Alamos National Laboratory strongly supports academic freedom and a researcher's right to publish; as an institution, however, the Laboratory does not endorse the viewpoint of a publication or guarantee its technical correctness. 
LA-14033

Issued: April 2003

The Induction of Chaos in

Electronic Circuits

Final Report — October 1, 2001

Robert M. Wheat Jr.

\author{
- Los Alamos \\ NATIONAL LABORATORY \\ Ideas That Change the World
}





\section{TABLE OF CONTENTS}

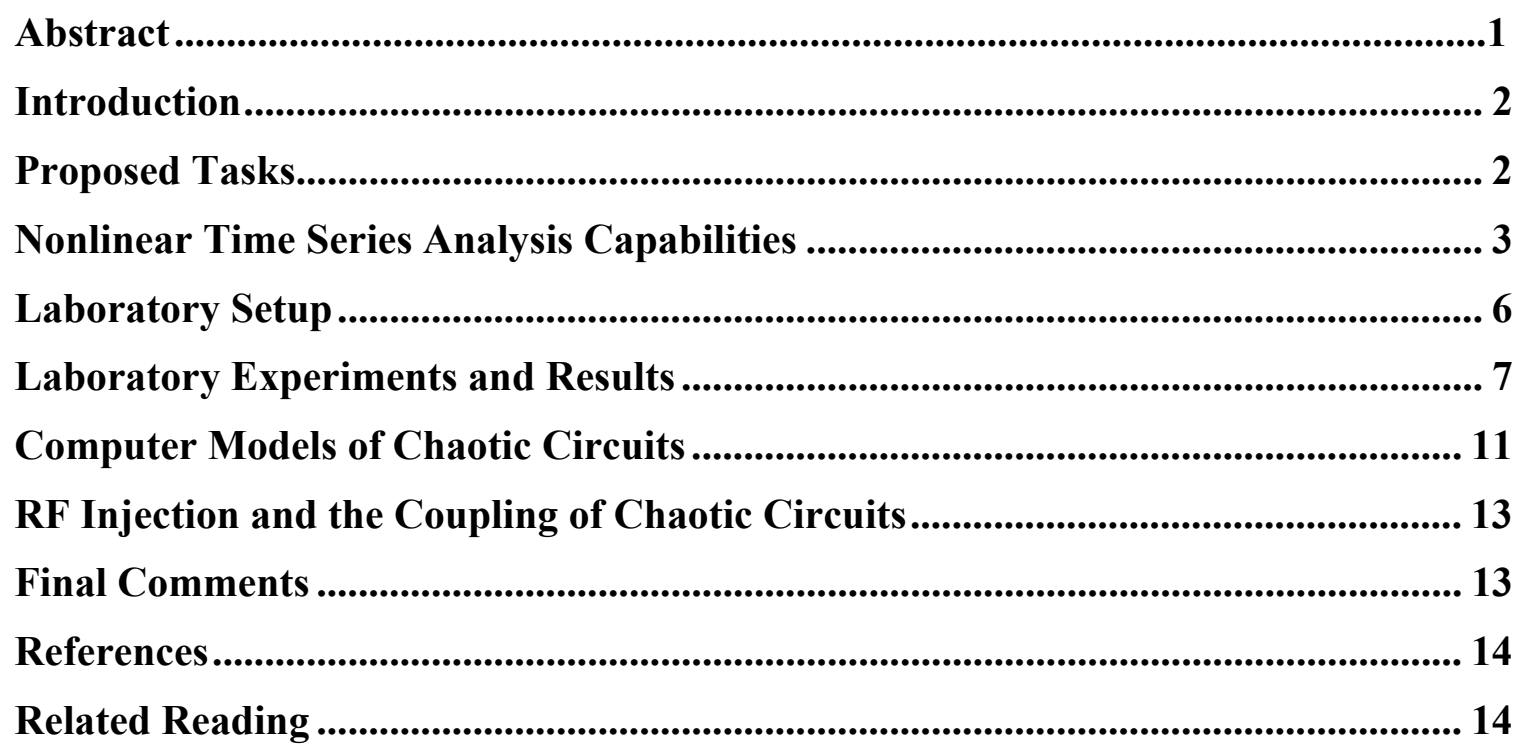


- vi - 


\title{
The Induction of Chaos in Electronic Circuits \\ Final Report - October 1, 2001
}

Robert M. Wheat, Jr.

\begin{abstract}
This project, now known by the name "Chaos in Electronic Circuits," was originally tasked as a two-year project to examine various "fault" or "non-normal" operational states of common electronic circuits with some focus on determining the feasibility of exploiting these states. Efforts over the two-year duration of this project have been dominated by the study of the chaotic behavior of electronic circuits. These efforts have included setting up laboratory space and hardware for conducting laboratory tests and experiments, acquiring and developing computer simulation and analysis capabilities, conducting literature surveys, developing test circuitry and computer models to exercise and test our capabilities, and experimenting with and studying the use of RF injection as a means of inducing chaotic behavior in electronics. An extensive array of nonlinear time series analysis tools have been developed and integrated into a package named "After Acquisition" (AA), including capabilities such as Delayed Coordinate Embedding Mapping (DCEM), Time Resolved (3-D) Fourier Transform, and several other phase space re-creation methods. Many computer models have been developed for Spice and for the ATP (Alternative Transients Program), modeling the several working circuits that have been developed for use in the laboratory. And finally, methods of induction of chaos in electronic circuits have been explored.
\end{abstract}




\section{The Induction of Chaos in Electronic Circuits \\ Final Report - October 1, 2001}

\section{Introduction}

This project, now known by the name "Chaos in Electronic Circuits," was originally tasked as a two-year project to examine various "fault" or "non-normal" operational states of common electronic circuits with some focus on determining the feasibility of exploiting these states.

Chaos in electronic circuits is a specific "non-normal" phenomenon and has been the main focus of these efforts. Chaos, in this context, is defined as "displaying or possessing a strong dependence on initial conditions." Stated another way, a small change in a system's initial conditions results in a large difference in its operation or state at some small time period after time zero. This "non-normal" operating mode is unwanted, in general, since the normal operation of electronic circuits relies on the ability to predict the state of the system at any time during its operation.

Identifying and verifying chaotic modes of behavior in electronic circuits are only two of the challenges faced in the examination of this phenomenon. To deal with these and other challenges, an extensive array of nonlinear time series analysis tools have been developed and integrated into a package named "After Acquisition" (AA), including capabilities such as Delayed Coordinate Embedding Mapping (DCEM), Time Resolved (3-D) Fourier Transform, and several other phase space re-creation methods.

The ability to model this behavior with computer codes is a good first step toward developing the ability to predict chaotic behavior, and both are among some of the additional challenges faced when conducting a study into the chaotic behavior of electronic circuits. Many computer models have been developed for Spice and for the ATP (Alternative Transients Program), modeling the several working circuits that have been developed for use in the laboratory, as well as some circuits that have not yet been tested in the lab.

\section{Proposed Tasks}

The tasks we proposed to complete during the duration of this two-year program are as follows:

1) Set up laboratory space and equipment for the performance of experiments.

2) Procure computer simulation and analysis capabilities.

3) Perform a literature search.

4) Develop computer models.

5) Develop simple circuits for use in laboratory experimentation.

6) Develop the ability to identify chaotic behavior.

7) Gain a trust in the computer models and develop an understanding of the underlying phenomena.

8) Develop an ability to predict chaotic behavior in circuits.

9) Study and experiment with RF injection as a means of chaos induction.

The tasks as listed above were all accomplished except for task \#8 and the second half of task \#7. Although many computer models have been developed, and each aspect of the models has been checked and verified, it is not clear that we have yet developed an understanding of the underlying phenomena as far as chaotic behavior is concerned. Chaotic behavior is certainly a nonlinear one, and it is obvious which components in any 
of the models are contributing to the nonlinearity of the behavior, but in order to better understand the intricacies involved, more study must be done. Although the current computer models do display chaotic behavior under the same configurations as the laboratory circuits, the predictive capabilities of these tools can be much improved with a better understanding of the underlying phenomena.

\section{Nonlinear Time Series Analysis Capabilities}

One of the first tools we acquired for the analysis of data from chaotically behaving circuits was The Chaos Data Analyzer [1]. This is an excellent tool for learning and displaying the fundamentals of time series data analysis where chaotic behavior is expected (nonlinear time series), and has served as a baseline for the development of our own analysis software.

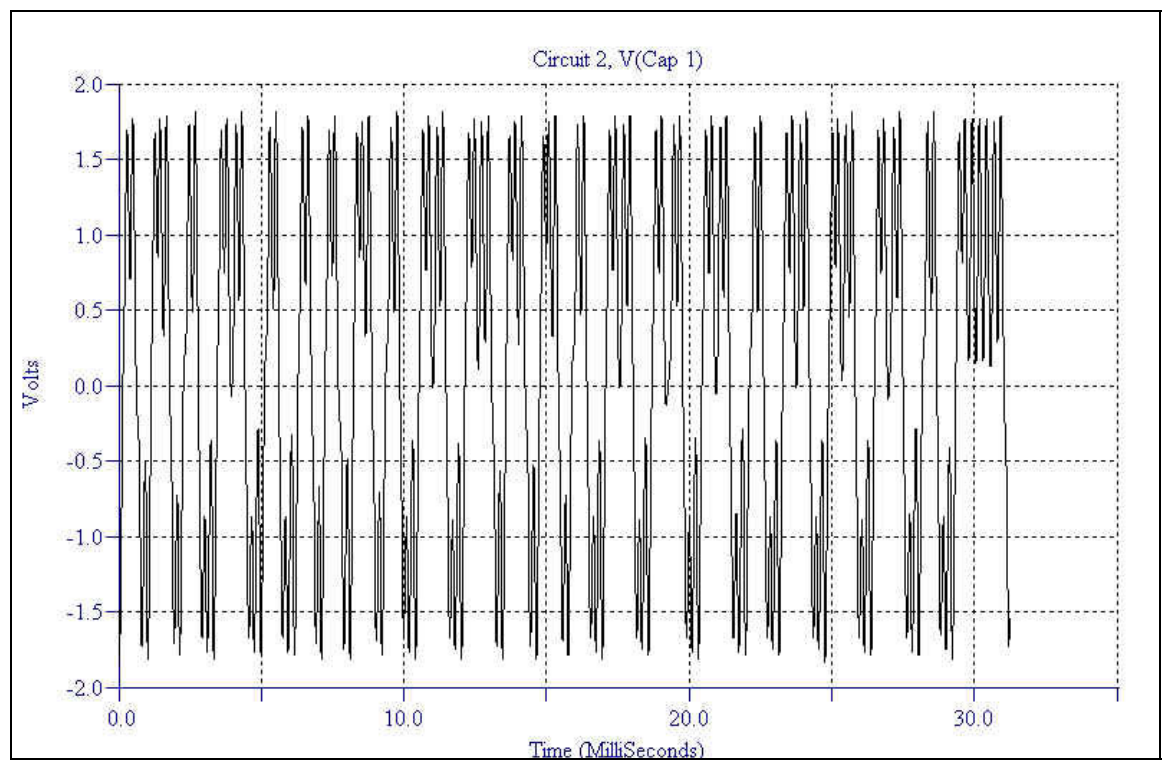

Figure 1. Time series data from an experimental electronic circuit.

The program we call AA is a program used to analyze time series data sets acquired from digital oscilloscopes or from circuit modeling software such as Spice or ATP. This software has been continuously developed over the course of this two-year project and has many fundamental capabilities of generic time series analysis software as well as capabilities unique to nonlinear time series analysis. Some of the fundamental generic capabilities of the software developed for this project include the following:

- The ability to read and write several different data file formats

- The ability to plot raw data to the screen or a printer (see figure 1)

- The ability to perform simple mathematical operations on either the $\mathrm{x}$-axis data or the y-axis data

- The ability to perform array mathematics

- The ability to transform time domain data to the frequency domain using either the Fast Fourier Transform (FFT) or a high resolution Discrete Fourier Transform (DFT).

Some of the nonlinear time series analysis capabilities of AA for use in phase space trajectory re-creation include the following:

- Plotting one amplitude data set versus a second from the same system (see figure 2)

- The Delayed Coordinate Embedding Map (DCEM)

- Plotting the derivative of a signal versus the original signal 
- Plotting the original signal versus a sinusoidal signal with a frequency chosen by the user.

We have also developed unique (to the best of our knowledge) algorithms for recreating the phase space trajectory plot in three dimensions using OpenGL ${ }^{\mathrm{TM}}$ programming.

The phase space trajectory plot is an important tool in nonlinear time series analysis but is not always conclusive when attempting to determine if a data set can be considered chaotic or not. For example, figure 2 is the phase space plot for an obviously chaotic signal. The fact that there are multiple attractors, as well as multiple paths between these attractors is conclusive. In addition, this phase space plot is very much like the well known "Lorenz Attractor." The phase space plot shown in figure 3 is not nearly as obviously chaotic as is that in figure 2. The data from which this plot is derived is, however, chaotic, and this can be determined by calculating the largest Lyapunov exponent (discussed in the next section).

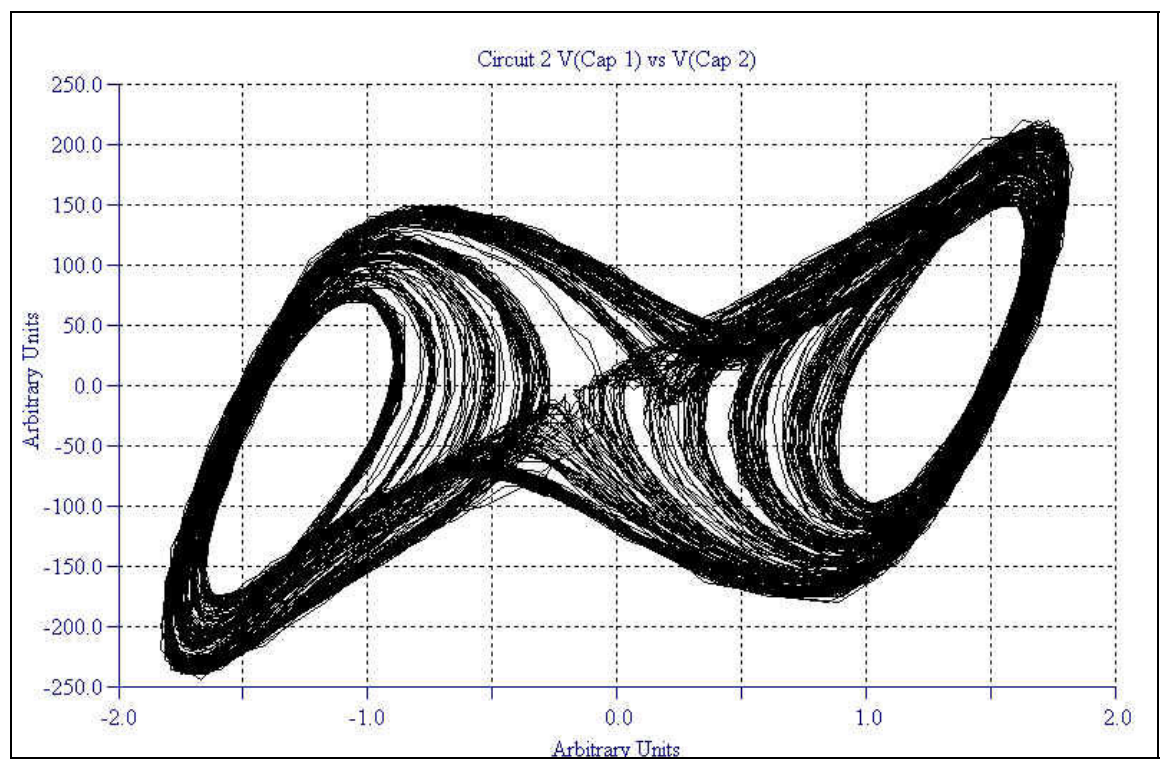

Figure 2. Phase space trajectory plot from an experimental electronic circuit.

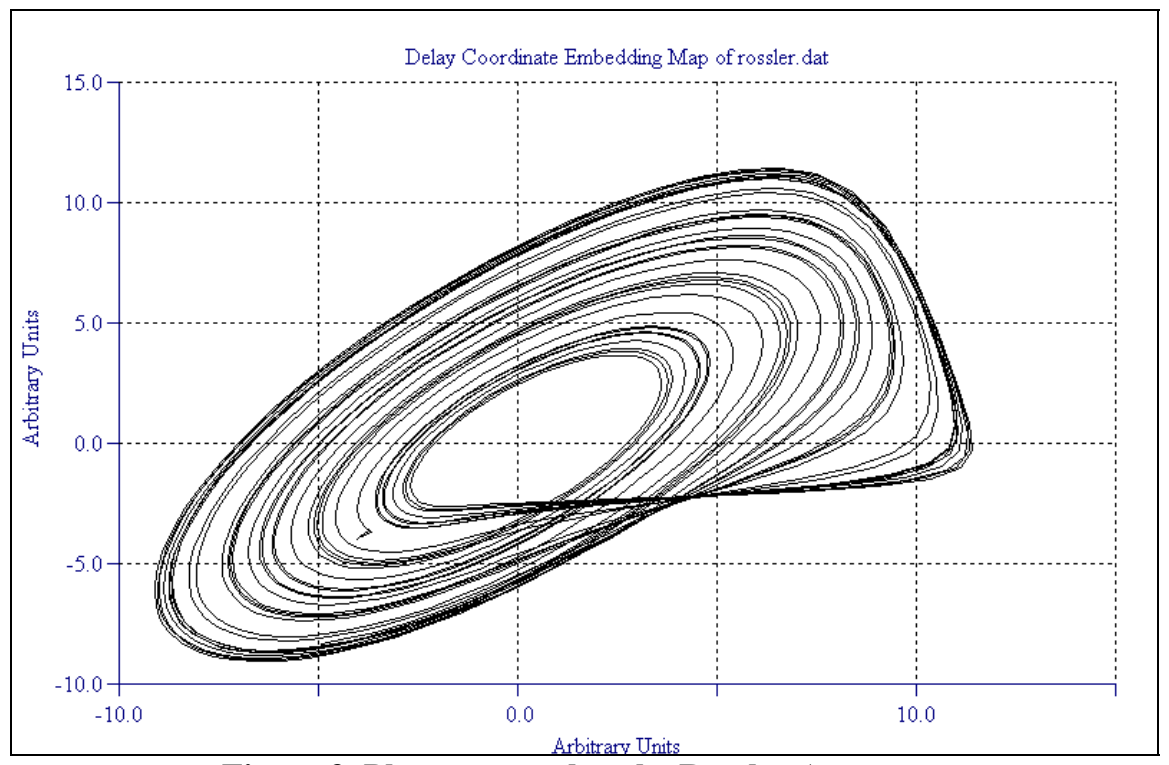

Figure 3. Phase space plot, the Rossler Attractor.

The software program AA already provides much functionality in the analysis of nonlinear time series, but much more needs to be done. The largest Lyapunov exponent, 
which is one of the few "litmus tests" for chaos, describes or quantifies the amount of chaos in a system. Technically, it is a measure of the divergence or convergence of trajectories in the state space of a system. In a nonchaotic system, the largest Lyapunov exponent will be zero or less than zero, and in a chaotic system where trajectory divergence will be exponential in some regions, the largest Lyapunov exponent will be greater than zero. The largest Lyapunov exponent, $\mathrm{S}(\Delta \mathrm{n})$, in a local small region, $\mathrm{U}\left(B \mathrm{n}_{0}\right)$, is given by the following:

$$
S(\Delta n)=\frac{1}{N} \sum_{n_{0}=1}^{N} \ln \left(\frac{1}{\left|U\left(\beta n_{0}\right)\right|} \sum_{\beta_{n} \in U\left(\beta n_{0}\right)}\left|\left(S n_{0}+\Delta n\right)-(S n+\Delta n)\right|\right)
$$

where $\mathrm{N}$ is the number of data points in the time series, $\beta \mathrm{n}_{0}$ is an arbitrarily chosen reference point or starting point, $\Delta \mathrm{n}$ is the number of time steps into the future to consider in the calculation, and $\left|\left(S n_{0}+\Delta n\right)-(S n+\Delta n)\right|$ is the length of the vector between the reference point and the current nearest neighbor of interest summed and averaged for all of the vector lengths from the $\mathrm{n}_{0}$ 'th time step to the $\mathrm{n}_{0}{ }^{\prime}$ th $+\Delta \mathrm{n}$ 'th time step [2, $\left.\mathrm{p} .63\right]$.

By performing this calculation on every "local small region" occupied by the phase space trajectory plot, one can determine if there exists an exponential divergence in the trajectory. If such a divergence exists, then the signal must be chaotic.

The implementation of the algorithm to calculate the largest Lyapunov exponent has begun, but much work in the form of testing and verification must be done.

In addition, several three-dimensional plotting algorithms have been developed and implemented using the OpenGL graphics programming language in the current version of the software. These functions provide unique capabilities and insights in the analysis of nonlinear time series, and as such, require much more testing and verification.

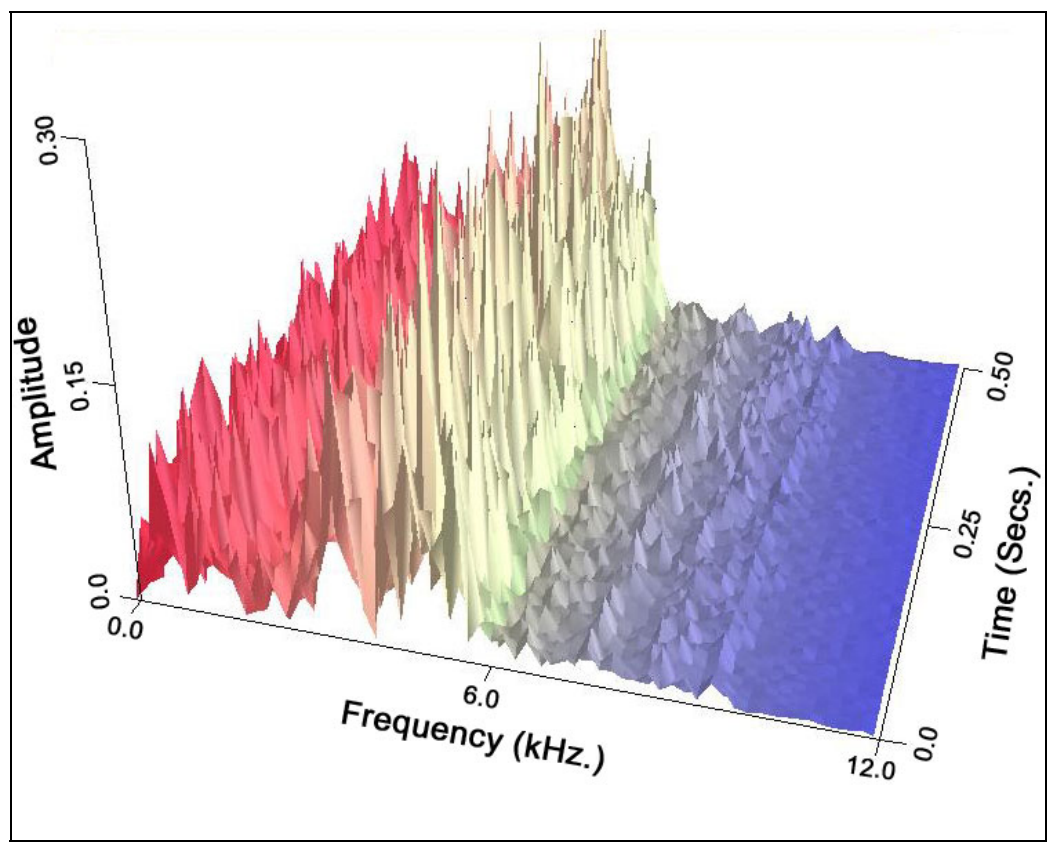

Figure 4. Time Resolved Fast Fourier Transform.

Figure 4 shows a time resolved Fourier transform. The program allows the user to rotate the plot around any or all axes as well as to zoom in and out. One of the characteristics of an electronic circuit behaving chaotically is the fact that a relatively wide band of frequencies are involved in the operation of the circuit, where in a "normally" operating resonant circuit, there is typically only one dominant frequency. 
For a circuit that may be behaving chaotically in a weak or intermittent manner, the time resolved Fourier transform makes for an excellent diagnostic tool.

Figure 5 shows a three-dimensional plot of the same phase space shown in figure 2 (as described earlier). The plot has been rotated such that the view shown is slightly behind and beneath the plot.

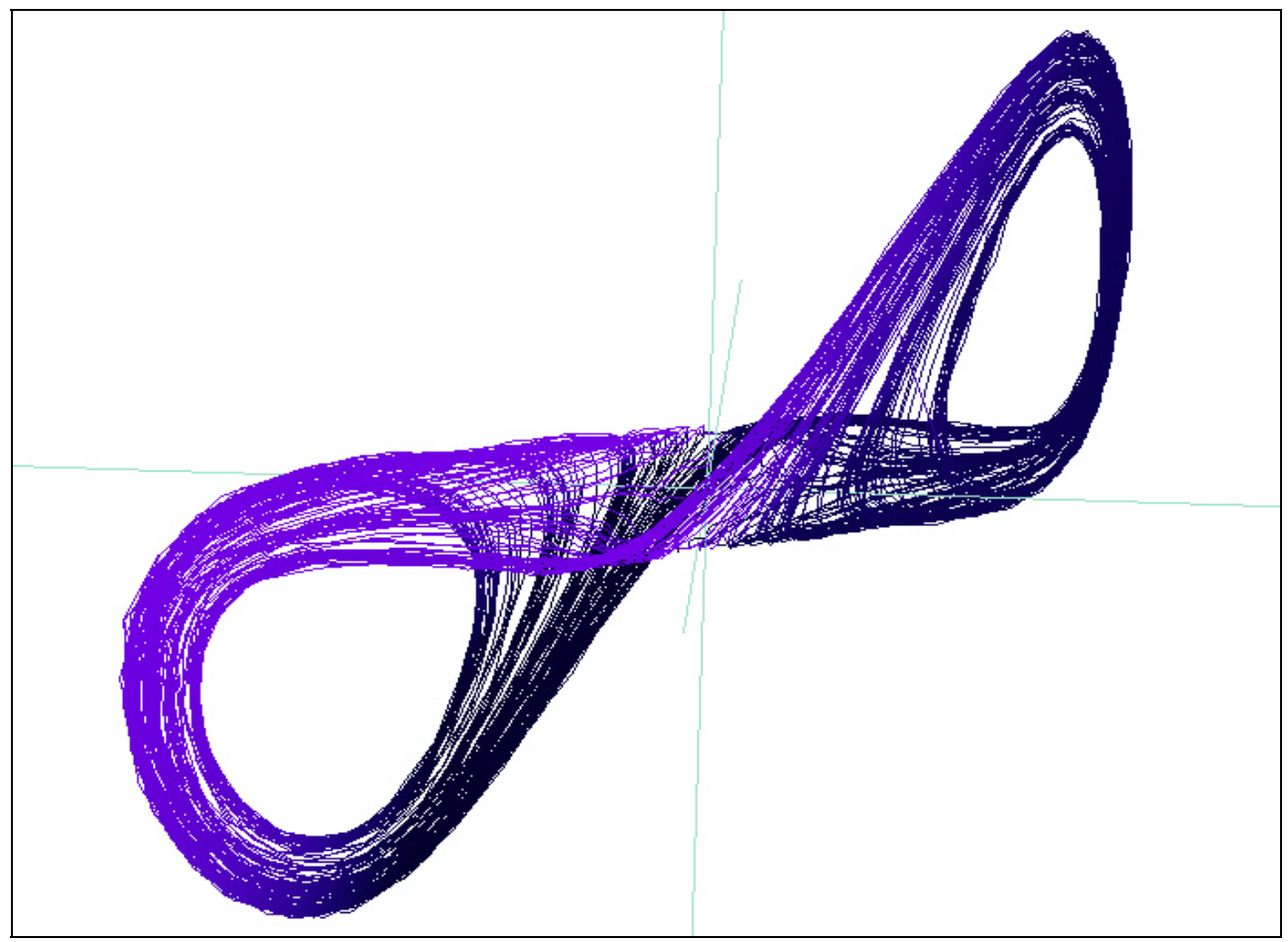

Figure 5. Three Dimensional Plot of the Phase Space for a Chaotic Circuit.

The following presents just a few of the many other useful algorithms that should be implemented in the software.

- Poincare maps

- Poincare flows

- Poincare movies

- Correlation matrix and dimension

- Bifurcation diagrams

- Capacity dimension.

It should be noted that the majority of the software developed in support of this program has been developed using tools under the Linux ${ }^{\mathrm{TM}}$ operating system. Some work has been done to 'port' this software to run under Microsoft ${ }^{\mathrm{TM}}$ operating systems, so as of the writing of this report, most software now works with MS Windows ${ }^{\mathrm{TM}}$.

\section{Laboratory Setup}

Laboratory space has been set up with appropriate laboratory equipment to perform experiments in support of this project. Three RF signal generators are part of the setup and have been used to explore the use of RF injection to induce chaotic behavior. In addition, two digitizing oscilloscopes have been employed as well as other laboratory equipment including, a power meter, a frequency counter, and a spectrum analyzer. Finally, a Dell $500 \mathrm{MHz}$ Pentium ${ }^{\mathrm{TM}}$ II computer, configured to triple boot Linux, MS Windows NT 4.0, and MS Windows 98, tops off the laboratory configuration for use in data acquisition, data analysis, and instrument control. Figure 6 is a photograph of the laboratory setup. 


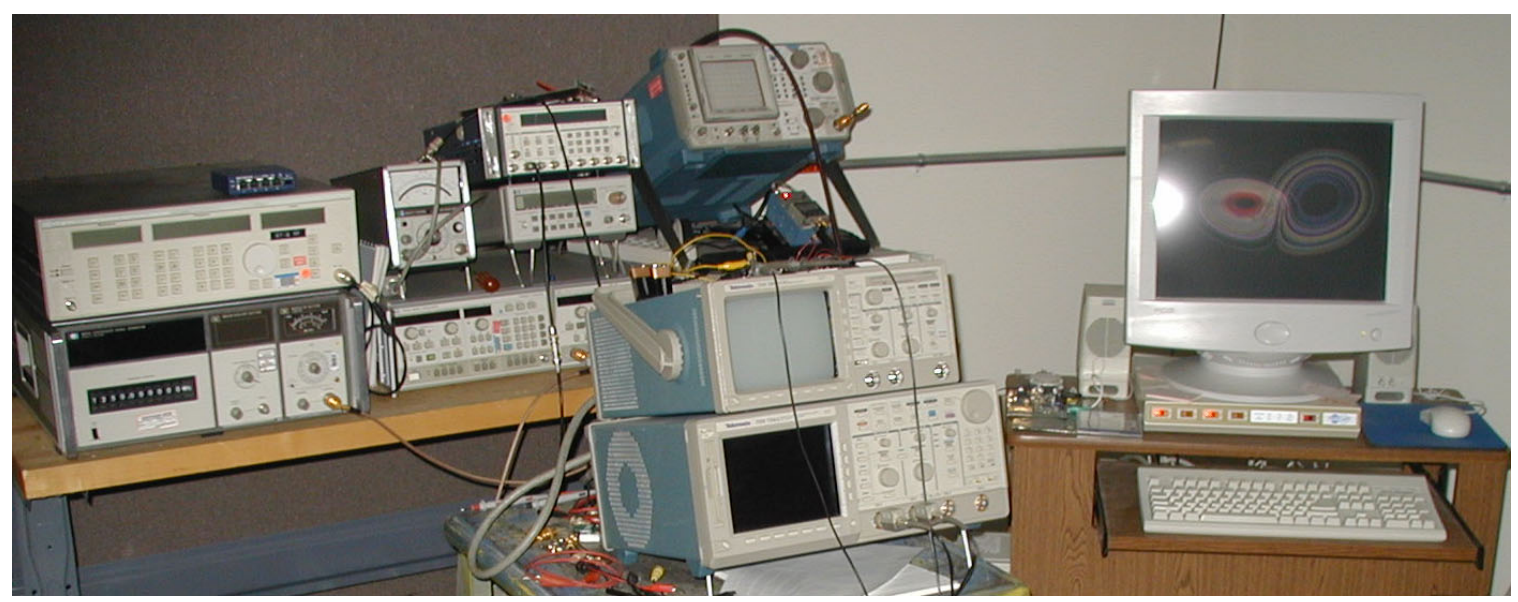

Figure 6. The laboratory setup.

\section{Laboratory Experiments and Results}

Initially, in order to develop our ability to identify and verify chaotic behavior in electronic circuits, we built a slightly modified version of a circuit originally designed by Leon Chua, which has already been proven to behave chaotically under known configurations $[3,4]$. The circuit is very simple, having only 1 I.C. (integrated circuit in the form of an operational amplifier), 1 inductor, 2 capacitors, 2 diodes, 8 fixed-value resistors, and one variable resistor (potentiometer) as its constituents. In this circuit, the diodes contribute the nonlinear properties necessary for chaotic behavior, while the I.C. acts as a negative resistance, feeding energy back into the circuit to maintain the oscillations when the circuit is appropriately configured as a resonant circuit. Figure 7 is a schematic of the circuit we have fielded in the laboratory, and figure 8 is a photograph of the actual bread board circuit.

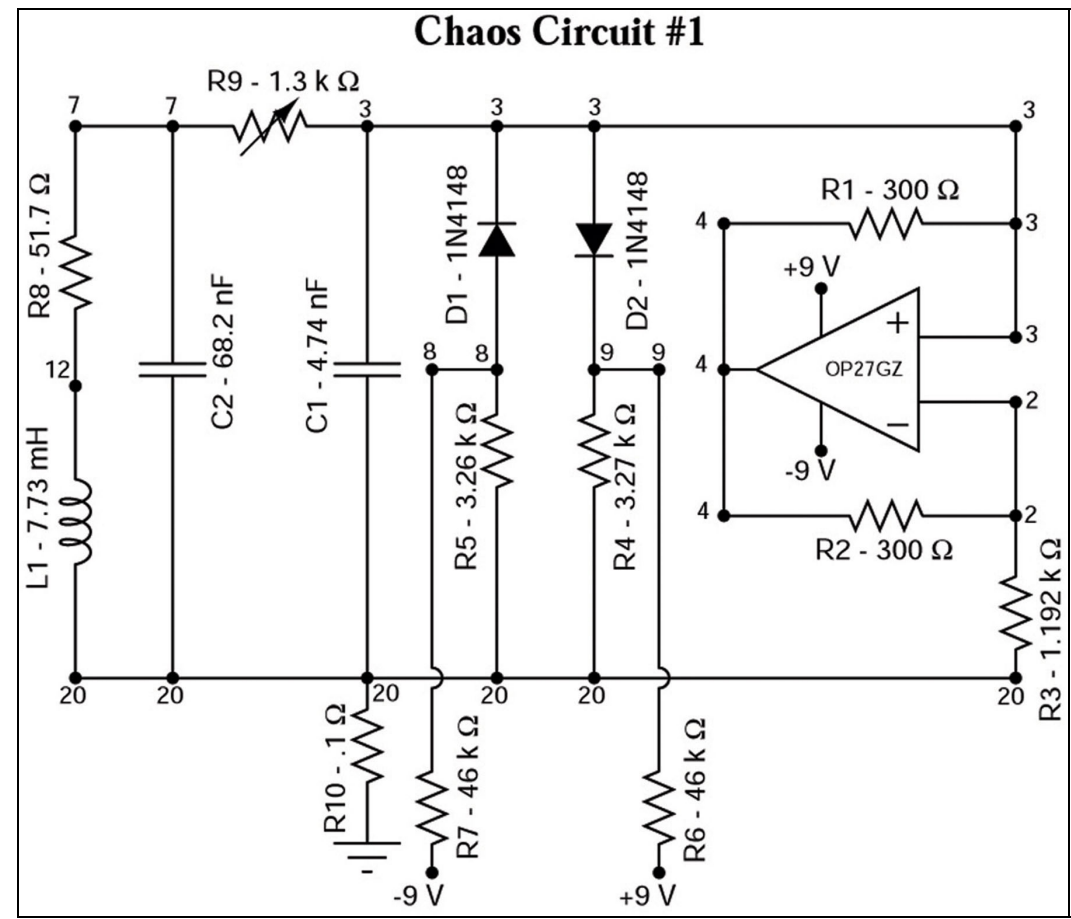

Figure 7. Schematic of the Fielded Circuit. 


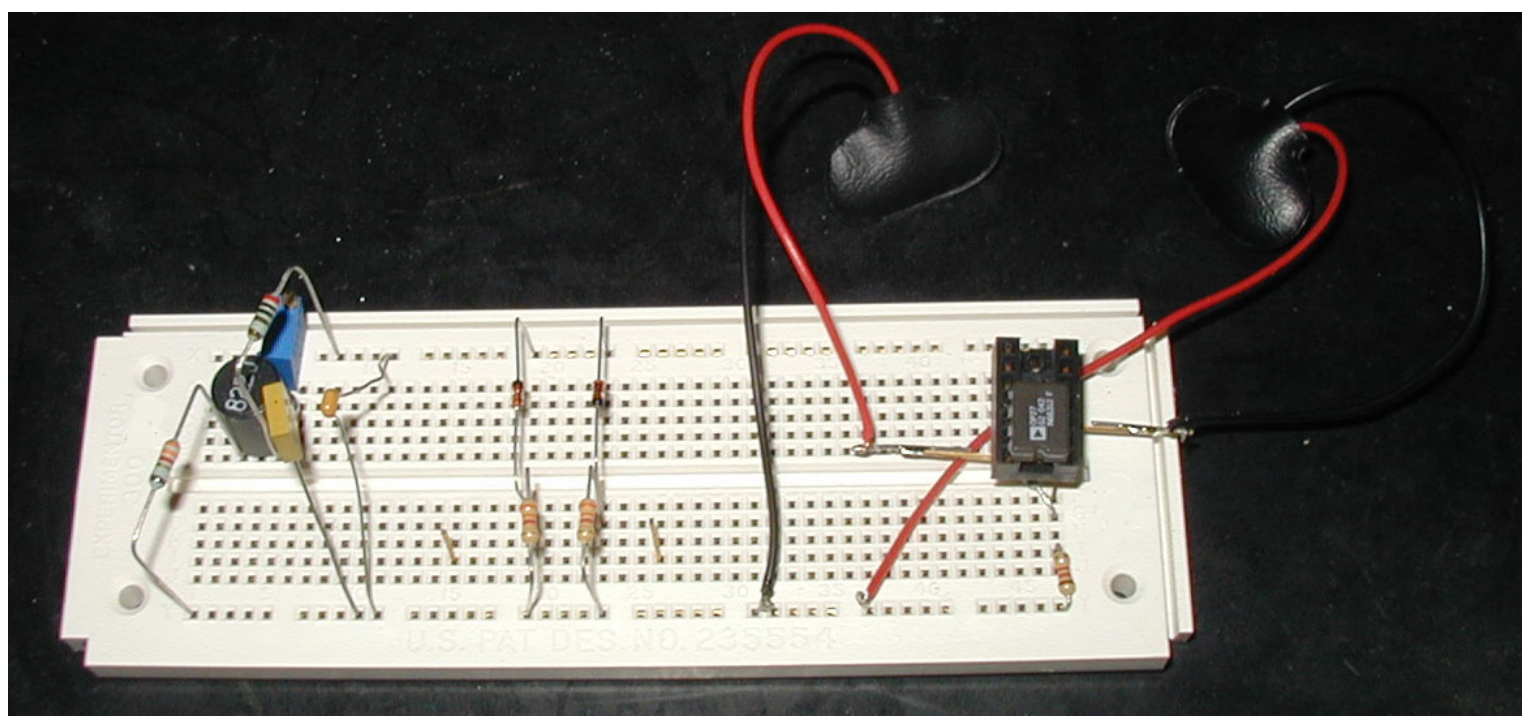

Figure 8. Photograph of One of the Actual Fielded Circuits.

This circuit is basically an LRC resonant circuit. When resistor \#9, the only variable component in the circuit, is adjusted to greater than $1295 \mathrm{ohms}$, then the circuit resonates, as one would expect, with a frequency of about $5.5 \mathrm{kHz}$. Increasing the value of this resistor further has little, if any, effect on the operation of the circuit. Figure 9 shows two plots. The plot on the left is the time domain response of our experimental circuit measuring the voltage across capacitor \#1. The plot on the right in figure 9 is the phase space trajectory plot re-created using the DCEM method and the time domain data. Normally, a sinusoidal signal would have a phase space trajectory that appears circular or elliptical.
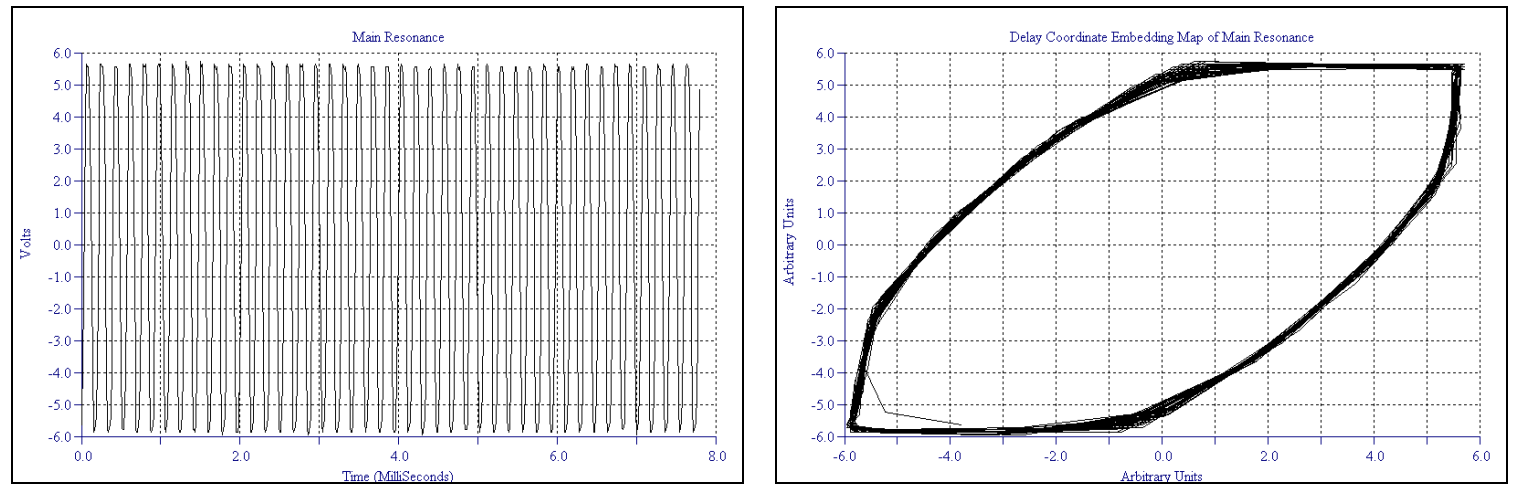

Figure 9. Main Resonance Mode; Left - Time Domain; Right - Phase Space Trajectory.
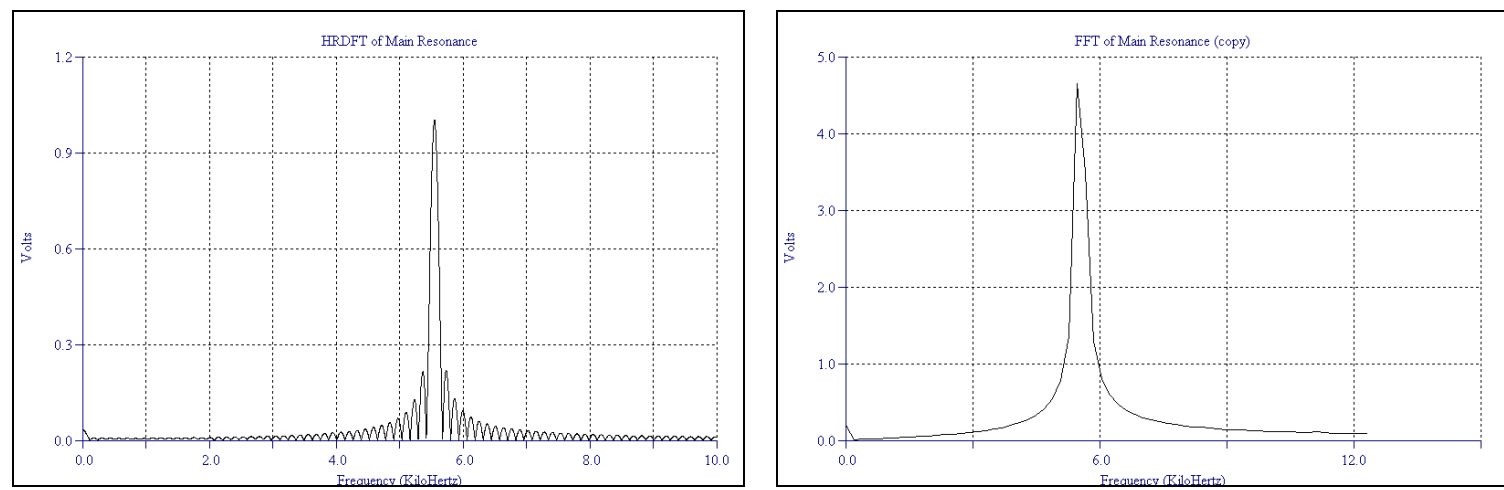

Figure 10. Main Resonance Mode Frequency Domain; Left - High-resolution DFT; Right - FFT. 
Figure 10 displays two frequency domain plots calculated from the raw time domain data. The plot on the left uses a high-resolution DFT algorithm developed by this author, while the plot on the right is the result of a typical FFT algorithm.

When the value of the variable resistor is adjusted below about 1275 ohms, the circuit stops oscillating altogether. As the resistance of this variable component is increased to about $1280 \mathrm{ohms}$, the circuit begins oscillating, but not like it was in its normal resonant mode, with the value of the resistor above $1295 \mathrm{ohms}$. This operational mode is what we will be referring to as "prechaotic."
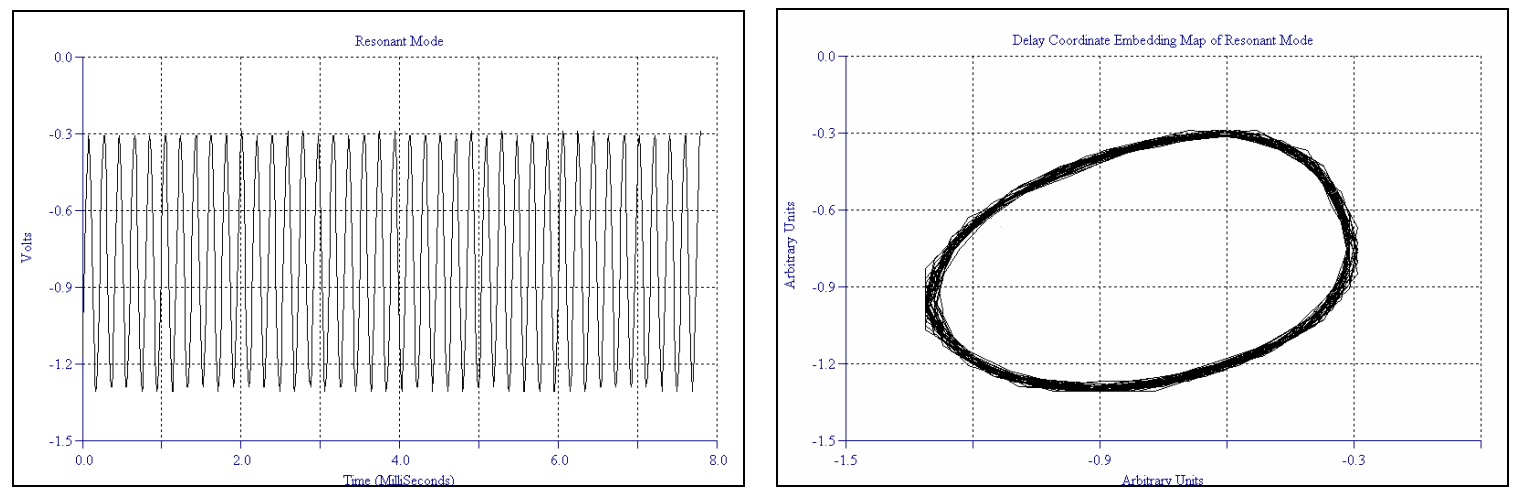

Figure 11. Pre-chaotic Operation; Left - Time Domain Data; Right - Phase Space Trajectory.

Figure 11 displays the time domain data, left, and the phase space trajectory, right, for the circuit operating in this prechaotic mode. Note that the time domain data is baseline shifted to the negative, causing the phase space trajectory to be completely in the third quadrant of a Cartesian system. The attractor now dominating the data is actually one of two possible attractors for this prechaotic mode. The second attractor is in the first quadrant of the Cartesian system. It is unclear what determines which attractor will dominate during this prechaotic operation, and in fact, if one observes this prechaotic mode for some period of time, the system will switch attractors. That is to say, the system, while operating in this prechaotic mode, switches back and forth between the two attractors on a time scale which is much greater ( 5 to 7 orders of magnitude) than the period of the resonance in the circuit.
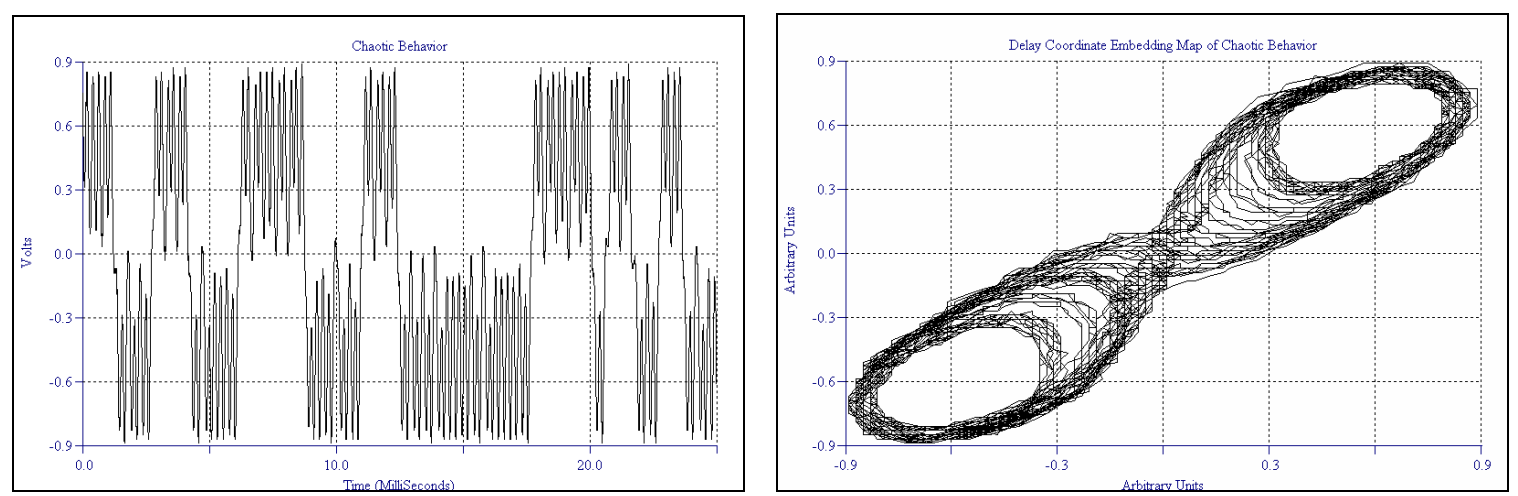

Figure 12. Chaotic Operation; Left - Time Domain Data; Right - Phase Space Trajectory.

As the resistance of the variable component is increased, the behavior of the circuit becomes chaotic. The system switches back and forth between attractors on a time scale that is roughly a factor of 1 - to 20 -times that of the resonance in the circuit. Figure 12, left, displays the time domain response of the circuit in voltage measured across capacitor \#1 while the circuit is operating in a chaotic manner, and right, displays the DCEM of the same. Figure 13 displays the frequency domain of the data acquired from the chaotically 
behaving circuit. Again, the left plot displays the frequency domain calculated using a high-resolution DFT algorithm developed by this author, and the right using a typical FFT algorithm. Of particular interest is the wide frequency band contributing to the chaotic signal. The $5.5 \mathrm{kHz}$ Fundamental is barely discernable as unique.

The development of this chaotic circuit has been a very important contribution to this project. It has allowed us to observe, firsthand, a circuit behaving chaotically. In addition, the development of this circuit has allowed us the ability to exercise our analysis algorithms and software on real data. Finally, the development of this circuit has afforded us a real-life circuit to compare our computer modeling results to. There will be a discussion of our computer modeling efforts later in this document.
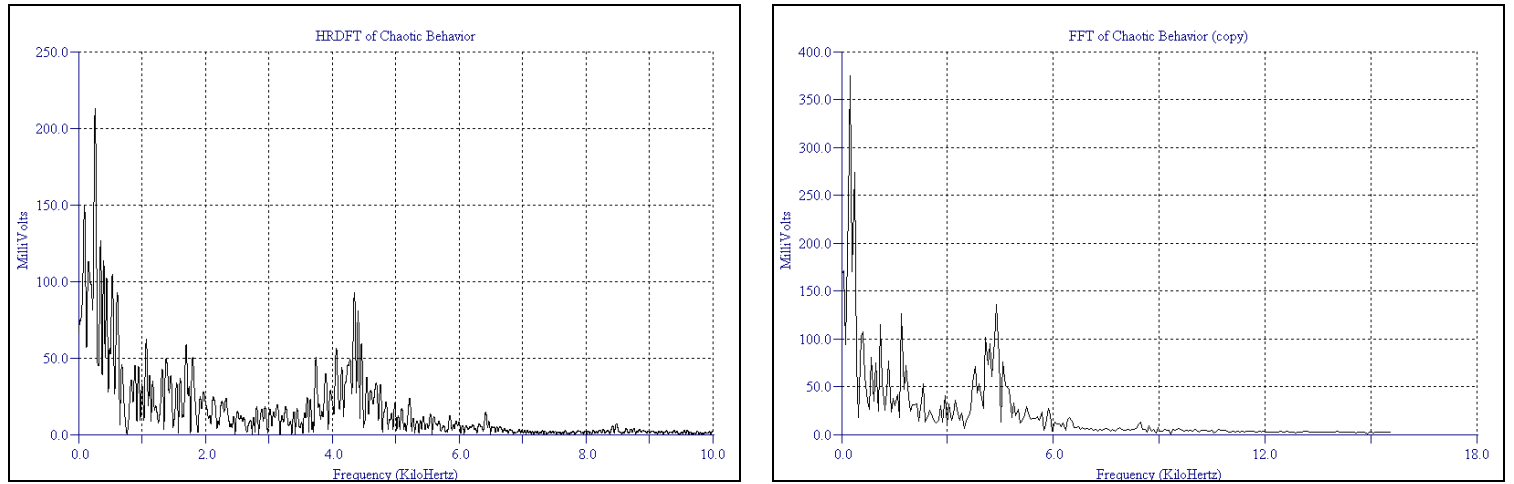

Figure 13. Chaotic Operation Frequency Domain; Left - High Resolution DFT; Right - FFT.

In addition to the chaotic circuit already mentioned, we have developed a demonstration circuit, "chaos in a box," powered by two nine-volt batteries, and special software. Using the voltages across each of the two capacitors in the circuit as input to a computer sound card, the software plots both the time domain data and the phase space trajectory in real time.

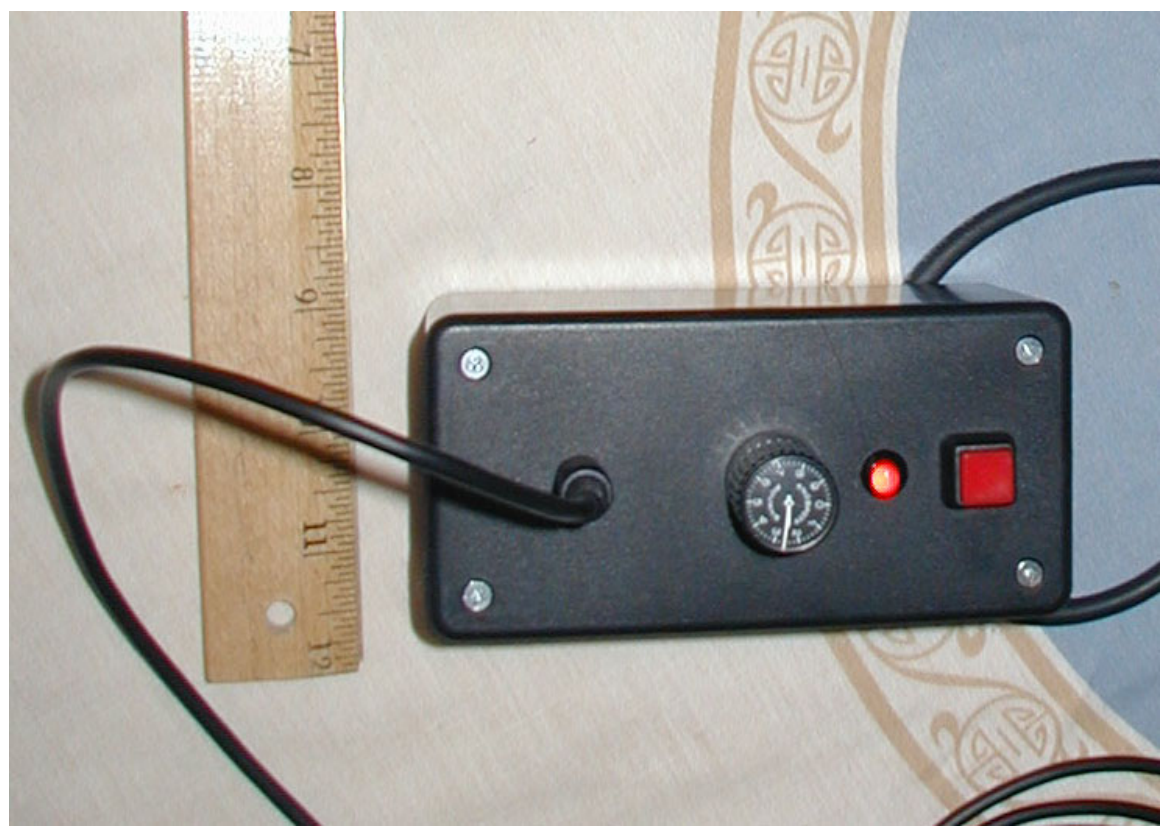

Figure 14. Chaos in a Box.

For the acquisition of data from the digitizing oscilloscopes, a command-line driven program has been developed for use with General Purpose Interface Bus (GPIB) interfaced scopes. This program has been developed using American National Standards 
Institute (ANSI) C standards so that it is portable between the Linux and MS Windows platforms. For the Linux platform, a Graphical User Interface (GUI) "wrapper" has been developed. Figure 15 is a picture of that interface. As the picture shows, the interface allows the user to choose the type of digital oscilloscope being used, as well as its GPIB address, which channels on that scope are being used, a disk drive directory and path for data file storage, and a common file name prefix. The program keeps track of the "shot number" and appends that as well as the channel number to the file name when saving the data.

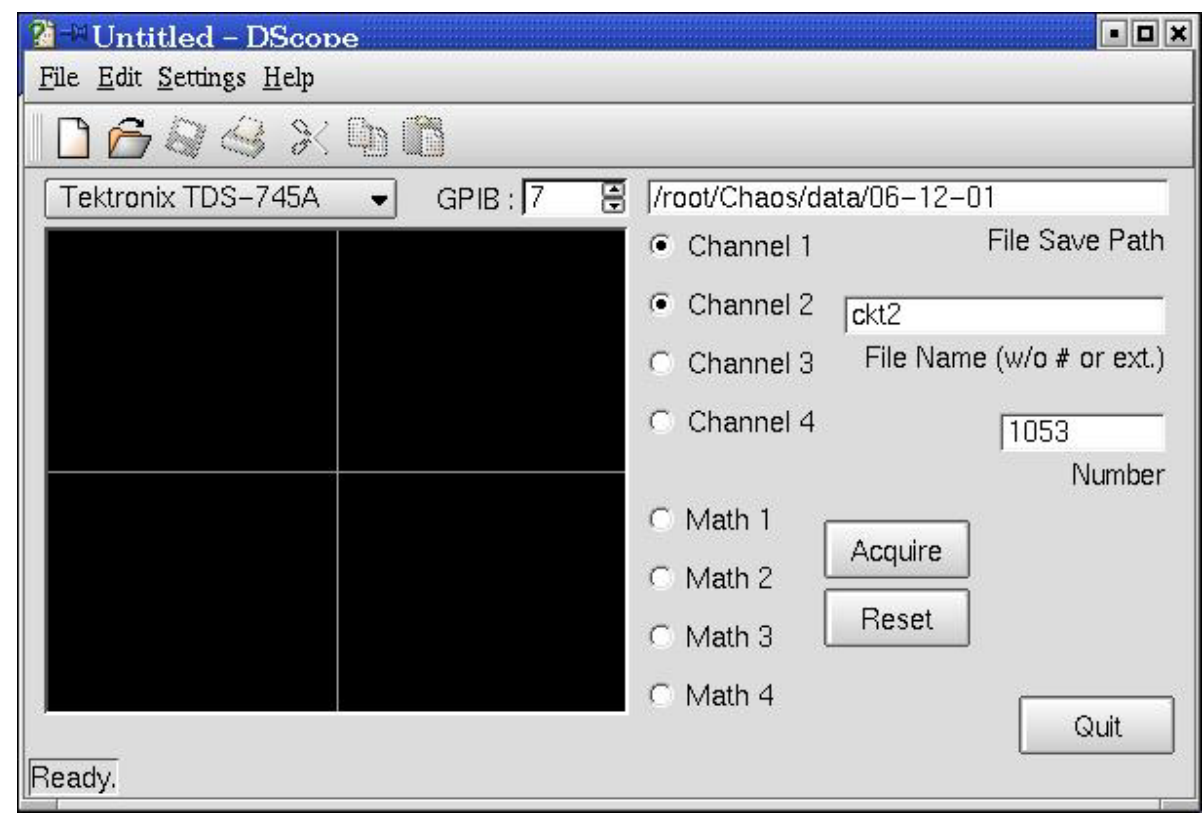

Figure 15. The Graphical User Interface for Data Acquisition.

\section{Computer Models of Chaotic Circuits}

Many Spice models have been developed in support of these efforts, including models for the op-amps diodes, as well as models for the actual circuits (see figure 7). The Spice models behave as expected and mirror the behavior of the actual circuits well. Figure 16 displays the time domain data, left, and phase space data, right, resulting from one Spice modeling run.
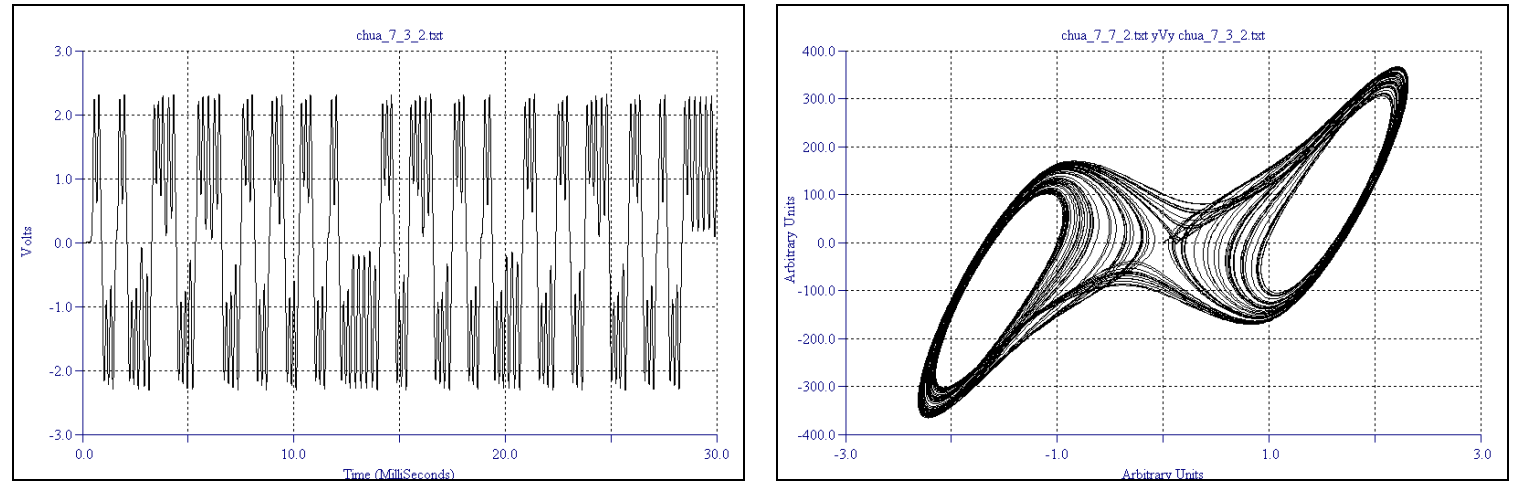

Figure 16. Spice Model Results; Left - Time Domain Data; Right - Phase Space Trajectory.

With some effort, computer models will prove to be an invaluable tool as far as the prediction of chaos in circuits. Many electronics manufacturers are now supplying Spice models to customers, and making use of these as well as the knowledge gained from the already developed models is an important next step. 
In addition to the Spice models, some of our recent efforts have shown the ability to model chaotic behavior in ferroresonant circuits with the ATP. Figure 17 (right) is a plot of the phase space trajectory from a run of one of our ATP models. As can be seen, a strange attractor is the obvious result. The fact that the attractor is "strange" does not automatically mean the system is behaving chaotically, but after calculation of the largest Lyapunov exponent (the value is greater than zero), it can be concluded, that in this case, the attractor is chaotic.
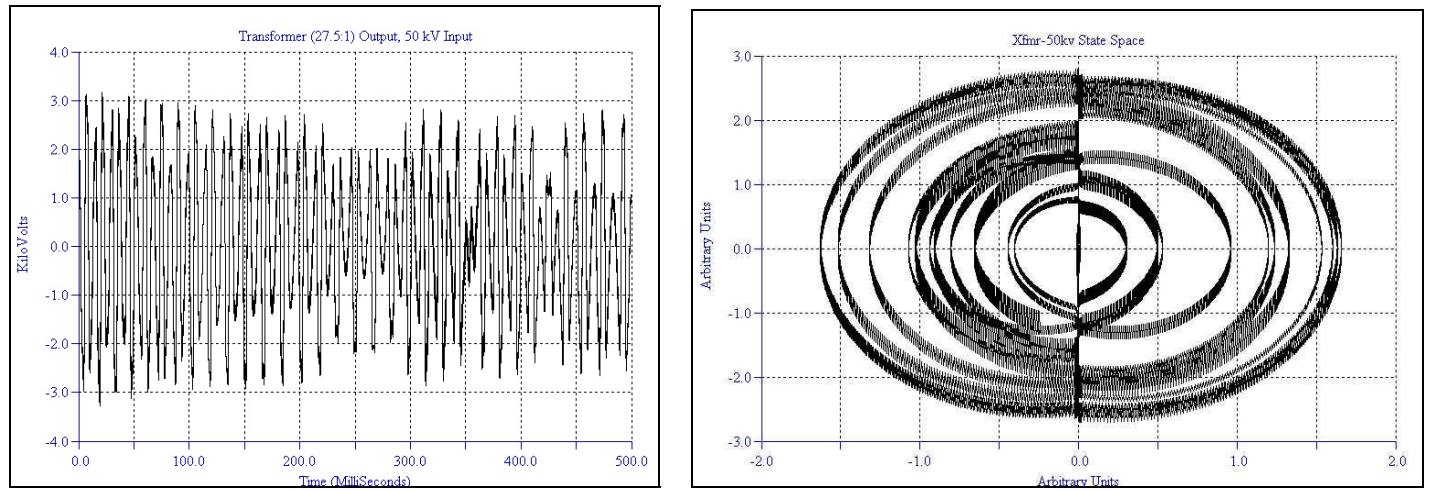

Figure 17. ATP Model Results; Left - Time Domain Data; Right - Phase Space Trajectory.

Ferroresonance is a rather destructive chaotic phenomenon that can occur in almost any ferroresonant circuit, specifically a circuit with an iron core transformer, under a variety of conditions, and induced by a variety of mechanisms. Some of the conditions and methods of induction of this phenomenon have been studied and documented over the past eight decades, but there is much that is still unknown concerning this phenomenon. In addition, the majority of the studies into ferroresonance have been focused on specific types of three-phase transformers, even though the models can be simplified to a single phase and have been conducted from the point of view of the power distributor, that is, prevention of this behavior $[5,6,7]$.

A basic ferroresonant circuit consists of a combination of a voltage source, a capacitance, and a nonlinear inductor (transformer) connected in series. Ferroresonant behavior typically consists of overvoltages and overcurrents of a factor of 1.5 to 5.0 or more whose waveform is highly distorted, and which can last for seconds to tens of seconds. This condition can be highly destructive to the control components in a power delivery system, the equipment fed by the system, and even the transformer itself.

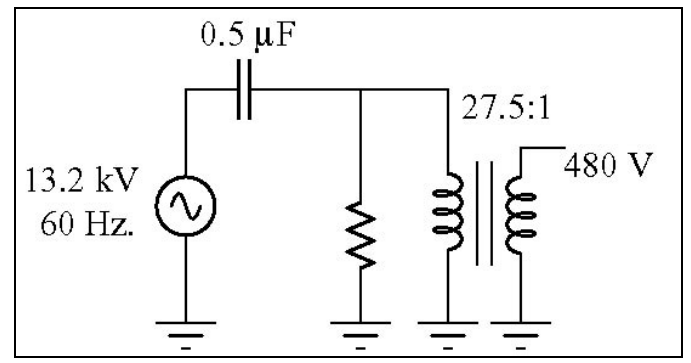

Figure 18. Schematic of the ATP Model.

Figure 17 (left) show a time series plot of the voltage waveform on the output side of a step-down transformer modeled with the ATP. The model is a simple single-phase circuit composed of a $13.2 \mathrm{kVrms}$ A.C. power source (18.7 $\mathrm{kVp}$ to $\mathrm{p}$ ), a $0.5 \mu \mathrm{F}$ capacitor, and a 27.5:1 transformer with a nonlinear inductor included as part of the transformer. A schematic of this simple model is shown in figure 18. During normal operation of this circuit, the output should be sinusoidal, peaking at about plus or minus 550 volts. 
The prospect of exploiting this ferroresonant behavior is very good, and the study of this should be done in parallel with the other studies into the chaotic behavior of electronic circuits.

\section{RF Injection and the Coupling of Chaotic Circuits}

Work began, during the two-year period of this project, to examine the use of RF injection as a means of inducing chaos in electronic circuits. No results worth reporting have been gained from these preliminary experiments, but based on these experiments and some reports acquired from our literature search, we have been able to formulate some ideas for further research.

The prospect that many circuits can be induced to behave chaotically can be exploited for various missions. For example, a circuit behaving chaotically can be coupled to another circuit (nonchaotic or chaotic) to draw it into behavior that is synchronized with the controlling chaotic circuit. Some work has already been done in the area of coupled chaotic circuits, but for different reasons than those discussed here. There is much work to be done in this area from the point of view of commandeering a target circuit [8].

We have begun investigating the range of conditions that will allow such take-over of a target circuit using a second Chua Circuit as the target circuit. Two similar circuits have already been assembled for this purpose. Basic work to characterize both the target circuit and the controlling circuit was underway as this two-year program came to a close.

\section{Final Comments}

In a recent report titled "Recent Developments in the Study of Nonlinear Phenomena in Power Electronics Circuits," author Chi K. Tse states "as the field of power electronics gains maturity and as the demand for better functionality, reliability, and performance of power electronics increases, in-depth analysis into nonlinear behavior and phenomena becomes justifiable and even mandatory."

Chaotic behavior of electronics has been ignored and "tuned out" for decades in the interest of getting electronic hardware and apparatus to the consumer in the most profitable manner (i.e., the shortest amount of time and least amount of man hours). Chaotic behavior in electronic circuits may well be a real and possibly crippling vulnerability in existing electronics. 


\section{$\underline{\text { References }}$}

1) J. Sprott, G. Rowlands, The Chaos Data Analyzer, The Professional Version 2.1, American Institute of Physics, Physics Academic Software, 1998.

2) H. Kantz, T. Schreiber, Nonlinear Time Series Analysis, Cambridge University Press, 1997, New York.

3) Cross, Michael C. "Chua's Circuit," http://www.cmp.caltech.edu/ mcc/chaos_new/Chua.html, 2000.

4) Chua, Leon O., "The Genesis of Chua's Circuit," $A E \ddot{U}$ vol. 46, no. 4, pp. 250-257, 1992.

5) C. Tse, "Recent Developments in the Study of Nonlinear Phenomena in Power Electronics Circuits," IEEE Circuits and Systems Society Newsletter, vol. 11, no. 1, pp. 14-21, 47, 48, March 2000.

6) B. Mork, D. Stuehm, "Application of Nonlinear Dynamics and Chaos to Feroresonance in Distribution Systems," IEEE Transactions on Power Delivery, vol. 9, no. 2, April 1994.

7) R.A. Walling, T.M. Compton, K.D. Barker, L.E. Zimmerman, "Ferroresonant Overvoltages in Grounded Wye-Wye Padmount Transformers with Low-Loss Silicon-Steel Cores," IEEE Transactions on Power Delivery, vol. 8, no. 3, pp. 1647-1660, July 1993.

8) N. Rulkov, "Images of synchronized chaos: Experiments with circuits," Chaos, vol. 6, no. 3, pp. 262-279, 1996. 


\section{Related Reading}

1) H. Kantz, "Time Series Analysis in Reconstructed State Spaces," Stochastics and Dynamics, vol. 1, no. 1, pp. 85-111, 2001.

2) N.H. Packard, J.P. Crutchfield, J.D. Farmer, R.S. Shaw, "Geometry From a Time Series," Physical Review Letters, vol. 45, no. 9, pp. 712-716, September 1, 1980.

3) T.S. Parker, L.O. Chua, "Chaos: A Tutorial for Engineers," Proceedings of the IEEE, vol. 75, no. 8, pp. 982-1008, August 1987.

4) T. Sauer, J.A. Yorke, M. Casdagli, “Embedology,” Journal of Statistical Physics, vol 65, no 3-4, pp. 579-616, 1991.

5) F. Takens, "Detecting Strange Attractors in Turbulence," Lecture Notes in Mathematics, no 898, pp. 366-381, Springer-Verlag, 1981.

6) I. Chiang, C. Liu, P.P. Varaiya, F.F. Wu, M.G. Lauby, "Chaos in a Simple Power System,” IEEE Transactions on Power Systems, vol 8, no 4, pp. 1407-1417, November 1993.

7) T. Yang, K. Bilimgut, "Experimental Results of Strange Nonchaotic Phenomenon in a Second-order Quasi-periodically Forced Electronic Circuit," Physics Letters A, vol 236, pp. 494-504, December 22, 1997.

8) J.P. Eckmann, S.O. Kamphorst, D. Ruelle, S. Ciliberto, "Liapunov Exponents From Time Series," Physical Review A, vol 34, no 6, pp. 4971-4979, December 1986.

9) M.T. Rosenstein, J.J. Collins, C.J. De Luca, "A Practical Method for Calculating Largest Lyapunov Exponents From Small Data Sets," NeuroMuscular Research Center and Department of Biomedical Engineering, Boston University, 44 Cummington Street, Boston, MA, 02215, November 20, 1992.

10) J.C. Sprott, "Simple Chaotic Systems and Circuits," American Journal of Physics, vol 68, no 8, pp. 758-763, August 2000.

11) W. H. Press, S. A. Teukolsky, W. T. Vetterling, and B. P. Flannery, Numerical Recipes in C: The Art of Scientific Computing, Cambridge U.P., Cambridge, 1993.

12) J.D. Meiss, "Frequently Asked Questions about Nonlinear Science, sci.nonlinear," ftp://amath.colorado.edu/pub/dynamics/papers/sci.nonlinearFAQ.pdf, September 2000.

13) M. Banbrook, G. Ushaw, S. McLaughlin, "Lyapunov Exponents from a Time Series: a Noise-robust Extraction Algorithm," Chaos, Solitons, \& Fractals, vol 7, no 7, pp. 973-976, 1996.

14) E. Olbrich, H. Kantz, "Inferring Chaotic Dynamics from Time-series: On Which Length Scale Determinism Becomes Visible," Physics Letters A, vol 232, pp. 63-69, July 21, 1997.

15) T. Schreiber, "Interdisciplinary Application of Nonlinear Time Series Methods," Physics Reports, vol 308, pp. 1-64, 1999.

16) A. Wolf, J.B. Swift, H.L. Swinney, J.A. Vastano, “Determining Lyapunov Exponents from a Time Series," Physica D 16, pp. 285-317, 1985. 
17) M.T. Rosenstein, P.R. Cohen, "Concepts From Time Series," Computer Science Department, LGRC, University of Massachusetts, Box 34610, Amherst, MA, 010034610, American Association for Artificial Intelligence (www.aaai.org), 1998.

18) A.G. Darbyshire, "Calculating Liapunov Exponents from a Time Series," Department of Physics, University of Oxford, Parks Road, Oxford, OX1 3PU, UK, The Institution of Electrical Engineers, 1994.

19) C. Grebogi, E. Ott, J.A. Yorke, "Attractors on an $N$-Torus: Quasiperiodicity Versus Chaos," Physica D 15, pp. 354-373, 1985.

20) C.B. Wallace, "Chaos in Simple Circuits at Microwave Frequencies: Simulation and Experiment," Information and Technical Strategic Business Unit, TRW, Alburquerque, NM, 87110, 199?.

21) A. Buermen, I. Fajfar, J. Puhan, T. Tuma, B. Zdralo, "Spice Opus: SPICE with Integrated Optimization UtilitieS," The University of Ljubljana, Slovenia, 2000, Georgia Tech Research Institute, 1994, Regents of the University of California, 1992. 
This report has been reproduced directly from the best available copy. It is available electronically on the Web (http://www.doe.gov/bridge).

Copies are available for sale to U.S. Department of Energy employees and contractors from:

Office of Scientific and Technical Information P.O. Box 62

Oak Ridge, TN 37831

(865) 576-8401

Copies are available for sale to the public from: National Technical Information Service

U.S. Department of Commerce

5285 Port Royal Road

Springfield, VA 22616

(800) 553-6847 
